\title{
Una etiqueta de plomo con fitónimos procedente de Mas Vell (Vallmoll, ager Tarraconensis)
}

\author{
A lead label with plant names from Mas Vell \\ (Vallmoll, ager Tarraconensis) \\ DIANA GOROSTIDI PI* \\ ISABEL RODÀ DE LLANZA*
}

\begin{abstract}
RESUMEN
ABSTRACT

Presentación de una nueva etiqueta de plomo hallada casualmente en una zona

rural del ager Tarraconensis. La pieza presenta dos líneas de texto en las que se ha podido reconocer la presencia de dos

fitónimos. La placa está provista de un apéndice lateral en el que se sitúa el agujero para el hilo o cuerda que la unía a

la vasija o contenedor. Este elemento resulta muy poco usual en este tipo de documentos epigráficos.

PALABRAS CLAVE

Etiqueta de plomo, instrumentum inscriptum, fitónimos, medicamento, ager

A new lead label has been found by chance in a rural area of the ager Tarraconensis. The piece has two writing lines with two plant names. The plate is provided with a lateral appendix with a hole for the filament that connected it to the vessel or box. This element is very unusual in this type of epigraphic documents.

Tarraconensis.

\section{KEYWORDS}

Lead label, instrumentum inscriptum, plant names, medication, ager Tarraconensis.
\end{abstract}

Recibido el 16 de septiembre de 2012. Aceptado el 1 de octubre de 2012

En agosto de 2011 apareció en un campo del término municipal de Valls (Tarragona), en la partida conocida como Mas Vell, una pequeña etiqueta de plomo con una inscripción latina en una de sus caras. Por aquél entonces me hallaba en Heidelberg, disfrutando de la cálida acogida del profesor Géza Alföldy en la esplén-

\footnotetext{
* Investigadora «Juan de la Cierva» del Ministerio de Economía y Competitividad. Institut Català d'Arqueologia Clàssica, PI. Rovellat, s/n, 43003, Tarragona. dgorostidi@icac.net.

** Institut Català d'Arqueologia Clàssica, PI. Rovellat, s/n, 43003, Tarragona. iroda@icac.net. Este trabajo se enmarca en el proyecto de investigación «La explotación y comercio de los recursos naturales en el N. de la Hispania romana: lapis, metalla, aqua (código: HAR2011-25011)», financiado por el Ministerio de Economía y Competitividad.
} 
dida biblioteca del seminario de Historia Antigua de la Universidad. Mientras trabajaba en su sala dedicada a la epigrafía, recibí por correo electrónico la foto enviada por nuestro común amigo Jordi López. Casi de inmediato, intercambiando juntos unas primeras impresiones, pronto percibimos la importancia del documento, que aun siendo modesto, representaba uno de los pocos ejemplares de etiquetas plúmbeas halladas en Hispania y no sólo en el territorio de la antigua colonia Tarraconense. Felices del hallazgo, le prometí una pronta edición a fin de que la pudiera incluir en su apartado del CIL dedicado a los instrumenta selecta de Tarraco a la que estaba dedicando una importante parte de la revisión del material epigráfico de la ciudad. Desgraciadamente, su inesperado fallecimiento tres meses después impidió que llegara a conocer los resultados preliminares de este estudio que en ocasión de su homenaje aquí presentamos.

\section{DESCRIPCIÓN DEL OBJETO}

La pequeña pieza metálica fue hallada casualmente ${ }^{1}$. Su forma es apaisada y rectangular, con una marca de flexión transversal en el centro que ha afectado parte de la inscripción, que quedaba, en consecuencia, en la cara interior de la etiqueta $^{2}$. Sus medidas son $5,7 \mathrm{~cm} \times 3,6$. Su lado izquierdo está recortado dejando en el centro un apéndice cuadrangular en el que se encuentra un agujero perfectamente redondo realizado probablemente con un grueso punzón. Esta especie de asidero se encuentra actualmente doblado hacia la cara interior y en contacto con la superficie de la cara inscrita (Fig. 1). Es característica de estas plaquitas la presencia de agujeros, ya que a través de ellos pasaba el hilo o cuerda con la que se sujetaban al objeto que acompañaban. Sin embargo, no es muy frecuente encontrar este tipo de elementos exentos, ya que en la mayoría de los casos se trata de perforaciones realizadas en uno de los ángulos de la superficie metálica rectangular, sin alterar la forma generalmente rectangular de las piezas metálicas ${ }^{3}$.

1 La pieza actualmente se encuentra en una colección particular. Agradecemos a su dueño las facilidades dadas para su estudio.

2 La pieza se encontró de hecho doblada por la mitad. Fue su descubridor quien al abrirla percibió las letras e inmediatamente cayó en la cuenta de que se trataba de un objeto antiguo. Conocedor de su importancia, se puso en contacto con los investigadores del ICAC, a quienes la prestó amablemente para su restauración, estudio y publicación.

3 Sobre este tipo de instrumentum inscriptum, destacan los trabajos de R. FREI-STOLBA, «Eine paläographische Bemerkung zu den Bleietiketten aus Oberwinterhur-Vitudurum», Epigrafica 47, 1985, 6570; cfr. ead. «Les etiquettes du plomb: des documents de l'ecriture au quotidien», M. CORBIER and J.P. GUILHEMBET (eds.), L'écriture dans la maison romaine, 2011, pp. 331-344; E. RÖMER-MARTIJNSE, Römerzeitliche Bleietiketten aus Kalsdorf, Viena 1990; G. PACl, «Etichette plumbee iscritte», Acta coIloquii epigraphici latini, Helsinki 1995, 29-40; M. FEUGĖRE, «L'instrumentum, support de l'écrit», en M. FEUGÈRE et P.-Y. LAMBERT (dir.), L'écriture dans la société gallo-romaine (Gallia 61), 2004, 53-65; L. BIZZARINI, "Quattro laminette plumbee da Altino», Annali del Museo Civico di Rovereto, XXI, 2005, 12113; M. LOVENJAK, «Roman lead tablet from Ribnica with an inscription in cursive writing», Bull. Instrumentum 21, 2005, 42-43; M. PASQUALINI et alii, «Fréjus, Forum lulii. Fouilles de l'Espace Mangin», en RAN, 38-39, 2005-2006, 283-341; I. RADMAN-LIVAJA, Les plombs inscrits de Siscia (Tesis Doctoral) Paris 2010 . 


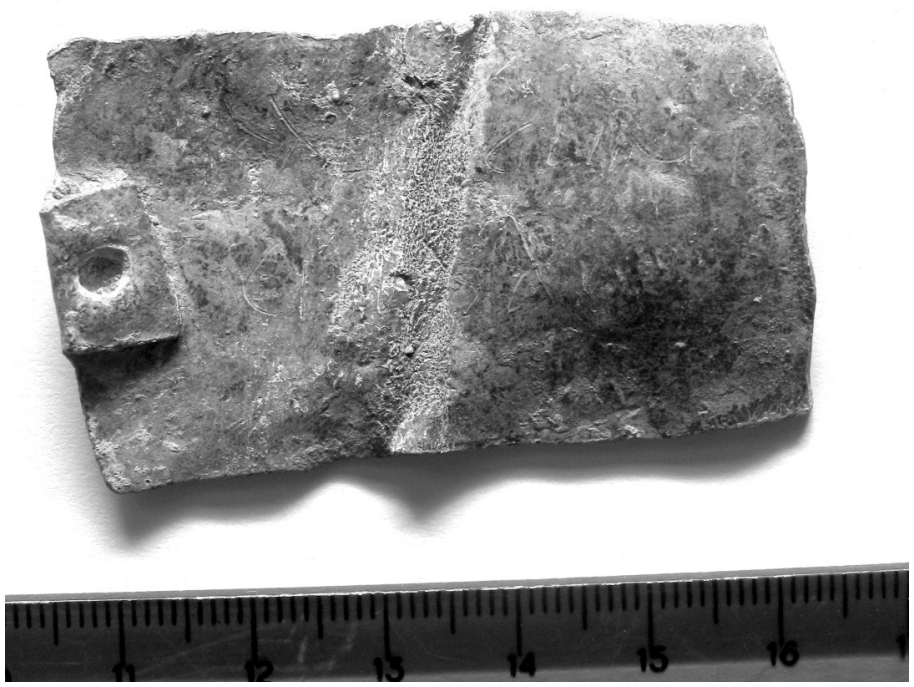

Fig. 1. Etiqueta de plomo de Mas Vell. (Foto: UDG / ICAC)

El texto fue inciso con un punzón de punta muy fina y se dispone en dos líneas paralelas. Las letras oscilan entre 1 y $1,5 \mathrm{~cm}$ y presentan los rasgos paleográficos de la minúscula cursiva romana, varias veces con un marcado carácter personal. Al contrario de lo que suele ocurrir, no presenta trazas de contener otros textos precedentes, ni tampoco su anverso presenta signos de haber alojado ninguna inscripción o marca.

La superficie conservaba una pátina que favorecía su lectura bajo óptimas condiciones de luz ${ }^{4}$. Tras la limpieza de la superficie, se procedió a realizar las fotografías con las que ha sido posible presentar una primera propuesta de lectura.

\section{DESCRIPCIÓN PALEOGRÁFICA}

La primera línea empieza con la secuencia PAS -con $\mathrm{P}$ inicial de panza completamente recta y diagonal, A de astil central inclinado y $S$ larga y estilizada. Sigue un trazo vertical conservado a media altura con un resto oblicuo en su lateral, a modo de T cursiva. La erosión producida por el doblez central ha afectado a la letra siguiente, de la que sobresale el trazo superior de una S larga muy estilizada. A continuación se aprecia el trazo curvo de una $\mathrm{C}$ o una $\mathrm{E}$ (de tipo griego) y dos astiles verticales, el primero de los cuales con una rebaba inferior que lo identifica como L, mientras el segundo puede ser leído como I, letra que también se en-

${ }^{4}$ Durante el proceso de restauración se llevó a cabo la asportación de la capa de cal mediante técnicas de abrasión, lo que ha afectado a la actual legibilidad del texto (sobre los riesgos del método, $\mathrm{PACl}$, op. cit., p. 32). Afortunadamente el registro fotográfico conserva una buena documentación de la pieza. 
cuentra en última posición. No obstante, antes de esta última I, es posible apreciar una grafía con un ductus a tres tiempos: una larga línea oblicua ascendente (1) a la cual se liga un trazo curvo horizontal (2) cuyo remate superior finaliza en un breve astil vertical descendente (3). Esta secuencia de trazos en la que el primero y tercero se unen mediante uno central sesgado puede ser interpretada como una $\mathrm{N}$, una forma que recuerda algunos ejemplos localizados en las tabellae Pompeianae. En el caso de la etiqueta que nos ocupa, aparecen los trazos segundo y tercero encadenados, a diferencia de los ejemplos campanos en los que a pesar de la proximidad se pueden reconocer los puntos donde se alzó el punzón. Esta scriptio continua quizá fuera favorecida por la mayor ductilidad del plomo respecto al estuco o la cera. Como se verá a continuación, el mismo fenómeno parece repetirse en la segunda línea con otra secuencia de trazos ${ }^{5}$.

La segunda línea inicia con las letras $\mathrm{Cl}$ bien reconocibles, seguidas de dos astiles verticales atravesados por la erosión producida por el pliegue central de la placa. En este lugar a duras penas puede apreciarse el resto de un tercer trazo vertical. Esta secuencia de tres astiles perpendiculares puede ser interpretada como IN en el supuesto de que el trazo oblicuo de la $\mathrm{N}$ se encontrara en la parte perdida correspondiente a la erosión del pliegue. En esta misma zona de desgaste se encuentra el arranque de un pequeño trazo oblicuo descendiente cruzado por otro superior más largo e inclinado en dirección contraria. Este segundo trazo continua hasta cerrar una secuencia de difícil interpretación paleográfica: una A de asta central oblicua o quizá la secuencia TI en ligadura, nexo ciertamente insólito ${ }^{6}$. Cierra la línea dos letras de clara lectura: una B del tipo llamado «à panse à gauche» y una I (Fig. 2).

PAST[-]SELINI

CI III++BI

\section{LECTURA E INTERPRETACIÓN}

Una vez descritos los caracteres tal como se presentan, pasamos a intentar dar una lectura en consecuencia. La primera línea presenta de una palabra iniciada por la secuencia pas- seguida probablemente de una $t$. La erosión central no permite identificar la letra que continua, pero el contexto reduce las posibilidades a pasta ${ }^{7}$. Pasta, -ae (del griego páste, -es), puede referirse tanto a la masa hecha de una o diversas sustancias machacadas, como a pasta, -ae, del copto pasté, un tipo de

5 Cf. Infra. Para el estudio paleográfico hemos utilizado los cuadros de tipos y los calcos publicados en los varios volúmenes de CIL IV, el estudio aún imprescindible de J. MALLON, Paléographie romaine, Madrid 1952, así como el cotejo de las fotografías publicadas en los diversos trabajos citados en la bibliografía recogida en las notas.

${ }^{6}$ Los modos de escritura en este tipo de documentos responden a usos muy particulares determinados por factores de diverso tipo, desde el nivel de pericia del escribiente a la ductilidad del soporte. Gianfranco Paci pone en evidencia que la mayúscula cursiva en las etiquetas de plomo se ejecuta «con molta libertà nella resa delle lettere» (PACl, op.cit, p. 32).

7 TLL, vol. X 1, p. 630. La S después de la laguna impide pensar en una palabra de más letras, por lo que las posibilidades quedan limitadas a la opción propuesta. 
envase o contenedor ${ }^{8}$. La elección de uno u otro significado dependerá de resto del texto.

A continuación, la secuencia de las letras siguientes resulta selini, interpretando la segunda letra detrás de la $\mathrm{S}$ como $\mathrm{E}$ y no como $\mathrm{C}^{9}$. Esta palabra parece emparentada con selinon, el nombre griego del apio (o perejil), un fitónimo bien documentado en las fuentes escritas. De hecho, aunque la forma latina más habitual para esta verdura sea selinas, -ados, también se documenta selinum ${ }^{10}$. Dado que a inicios de la segunda línea se puede leer claramente $\mathrm{Cl}$, es posible pensar en una forma selinicus, no documentada, aunque posible gracias al empleo del sufijo -icus usual para la formación de adjetivos ${ }^{11}$.

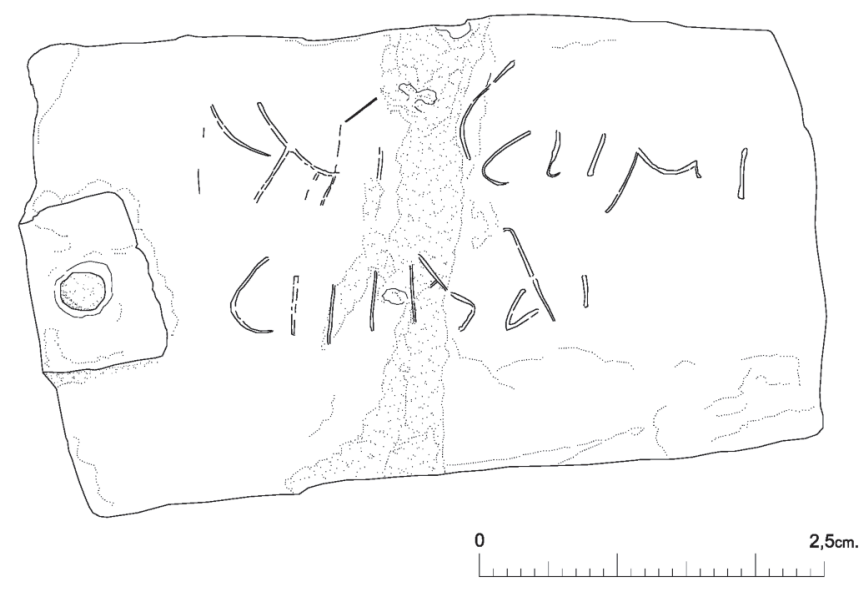

Fig. 2. Etiqueta de plomo de Mas Vell. (Dibujo: P. Aliende/ICAC)

El resto de la segunda línea contiene seis caracteres, de los cuales sólo los dos últimos son de lectura clara: BI. De las opciones propuestas en la descripción paleográfica, parece difícil encontrar una secuencia coherente a un final -abi (que remite a un nominativo -abus o -abum) precedida de los tres astiles verticales ${ }^{12}$. Sin embargo, una vez descartada la A, este final -bi puede permitir leer la segunda opción, aunque paleográficamente extraña: -tibi. A su vez, la sucesión de asti-

8 Documentado en un papiro copto (PLond. 2.191.9), datado en el siglo II dC. Con esta acepción aparece en el De medicamentis de Marcellus Empiricus (s. IV-V dC): mittes in pastam et deinde mittes in furnum $(30,49)$.

${ }^{9}$ La C queda descartada porque la secuencia SCL resulta infructuosa, así como también SCIL no parece adecuarse a las opciones de las letras restantes. Cf. Infra.

10 Para selinas, cf. OLD. Para selinum, cf. TLL, vol. X 1, p. 1983.

11 Cf. la forma adjetiva griega es selininos.

12 Las varias opciones pueden ser comprobadas gracias al Lewis-Short Latin Dictionary consultable online. Descartamos el topónimo Genabum (referido a la antigua Orléans), por ser su forma adjetivada Genabensis. 
les verticales a inicio de palabra pueden ser interpretados como in-, dando como resultado la forma en genitivo de otro fitónimo, intibis ${ }^{13}$. Se trata de la achicoria (cichorium intibus), que varios autores registran como remedio para dolencias estomacales ${ }^{14}$. La forma está bien documentada tanto en las fuentes latinas (Virgilio, Ovidio, Horacio, Columela, Plinio...) como en la epigrafía, ya que aparece registrado en el edicto de pretiis de Diocleciano (intiba optima) ${ }^{15}$. La identificación de esta planta junto con la forma selinicus referida al perejil o apio, plantas también utilizadas en la farmacopea antigua, así como la propuesta inicial de lectura past[a] consienten un contexto común a los tres elementos: la realización de una mixtura de carácter medicinal elaborada a partir de extractos de hierbas ${ }^{16}$.

La lectura, en consecuencia, es la siguiente:

\section{Past[a?]selini-/ci intibi.}

Traducción: «Preparado de achicoria y apio».

Se trata, pues, de una etiqueta de plomo que acompañaba probablemente una vasija que contenía este preparado. Tanto si se interpreta pasta como contenedor o como el medicamento mismo, el empleo de la palabra en este tipo de documentos resulta en ambos casos oportuno.

\section{EL SOPORTE}

Por su parte, la misma etiqueta como soporte merece un comentario. La forma de la placa es rectangular, como por lo general este tipo de documentos. Lo más destacable es, no obstante, el apéndice situado en el lado corto izquierdo, perfectamente recortado y con el agujero bien centrado en el pequeño espacio cuadrado resultante. En la mayoría de las etiquetas de este tipo tienen una perforación realizada dentro del área rectangular, las más veces en coincidencia con uno de los ángulos (Fig. 3) ${ }^{17}$. También la calidad de la escritura denota interés y cuidado. La

${ }^{13}$ Cf. ThLL, vol. VII 2, p. 14. La forma griega intybos o entybos resulta préstamo de la latina, de la cual deriva etimológicamente nuestra palabra endivia, aunque actualmente no corresponda con la misma especie vegetal.

${ }_{14}$ Por ejemplo, Dioscorides lo aplica para problemas gástricos y digestivos y es recomendado por Galeno para las dolencias de hígado. Cf. ThLL, op.cit., p. 16.

${ }_{15}$ M. GIACCHERO, Edictum Diocletiani et collegarum de pretiis rerum venalium, in integrum fere restitutum e Latinis Graecisque fragmentis, Genova 1974 (CIL III, p 2208, $2209=$ CIL XII, *69 = ILS 642). Para la forma, cf. ThLL, op.cit.

${ }^{16}$ Los vegetales son muy corrientes en los textos de este tipo de inscripciones. Por ejemplo, se ha documentado basilicum, allicium, piper, corfex (PACI, op. cit. p. 32) o asparagus (L. SCHWINDEM, «Asparagus - romischen spargel. Ein ueues Bleietikett mit Graffiti ans Trier», Funde und Ausguabungen in Bezirk Trier, 26, 1994, pp. 25-32). Para los usos medicinales de éstas y otras hierbas en la antigüedad, cf. Dioscórides / Pseudo Dioscórides, Plantas y remedios medicinales. Obra completa. Madrid 1998.

17 Como las etiquetas de Fréjus (PASQUALINI et alii, op.cit., con foto en página 319), Altino (BIZZARINI, op.cit.) o Siscia (RADMAN-LIVAJA, op.cit.), por citar algunos casos. A modo de ejemplo, un buen repertorio con fotografías del material del museo de Zagreb se puede ver en I. RADMAN-LIVAJA, «Le rôle des étiquettes de plomb dans le travail du textile à Siscia», en C. ALFARO et alii (eds.), Purpureae Vestes. III Symposium Internacional sobre Textiles y Tintes del Mediterráneo en el mundo antiguo, p. 183 fig. 1. 
ausencia de abreviaciones, tan características en estos documentos destinados a una función efímera como simples identificadores, ya sea del destinatario, contenido o precio, también indica una atención puesta por el productor también en el etiquetado de sus productos.

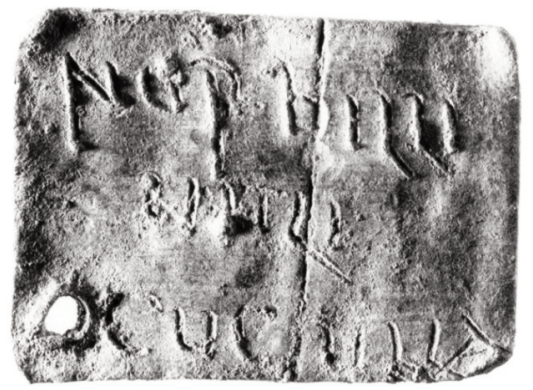

Fig. 3. Etiqueta de plomo de Fréjus (Francia). (Foto: ${ }^{1}$ hr. Durand, CNRS-CCJ)

De hecho, la presencia de este apéndice se puede identificar en otra plaquita de plomo hallada en Talairan, cerca de Narbona (Francia). Posee unas medidas y forma semejantes, dotada también en el lateral izquierdo del recorte cuadrangular, actualmente roto. El texto se reduce a una sola palabra: malaci, quizá del griego malakon («dulce»), o bien de la ciudad de Malaca (Malaci(tanus)?), referido al desconocido producto que acompañaba ${ }^{18}$. Aunque el apéndice fue en algún momento doblado y posteriormente arrancado, aún se puede apreciar el resto curvado de la pestaña, que asemeja tanto al ejemplar que nos ocupa (Fig. 4). Esta característica confiere a ambos soportes un particular modo de realización: quien diseñó la forma de la placa de plomo la dotó expresamente de una extremidad destinada a alojar el orificio en el cual atar el hilo de sujeción, lo que supone un elemento de sofisticación respecto a las etiquetas al uso y, en consecuencia, de intención en la elección del soporte, a los que hemos de sumar la escritura in extenso, rasgos de los que carecen en general el resto de etiquetas de plomo conocidas.

La ausencia de contexto arquiológico impide una datación más precisa que la genérica para este tipo de objetos, que por criterios paleográficos podemos situar en los dos primeros siglos del imperio ${ }^{19}$.

No abundan en Hispania las etiquetas de plomo: de hecho, la única que hemos documentado fue hallada en 1948 en Ampurias y está fechada a finales de la república $^{20}$. Se trata de un fragmento de etiqueta de plomo de pequeñas dimensiones con inscripción. Presenta como es habitual un orificio en uno de sus extremos y la

18 Corresponde a la ficha PIS-4001 extraída de la espléndida página web Artefacts. Encyclopédie en ligne des petits objects archéologiques (http://www.instrumentum-europe.org/Artefacts/home.php). Queremos agradecer al prof. M. Feugère la amabilidad de dejarnos publicar esta placa de plomo inédita.

19 PACl, op. cit., p. 33.

20 IRC III, 178 (con bibliografía precedente). 
lectura, aunque fragmentaria, permitía reconocer un preparado medicinal, ptarmicus, una substancia utilizada para estornudar, así como el inicio de un nombre propio, Phaon (?), identificado con la persona que elaboró tal remedio (Fig. 5) ${ }^{21}$.

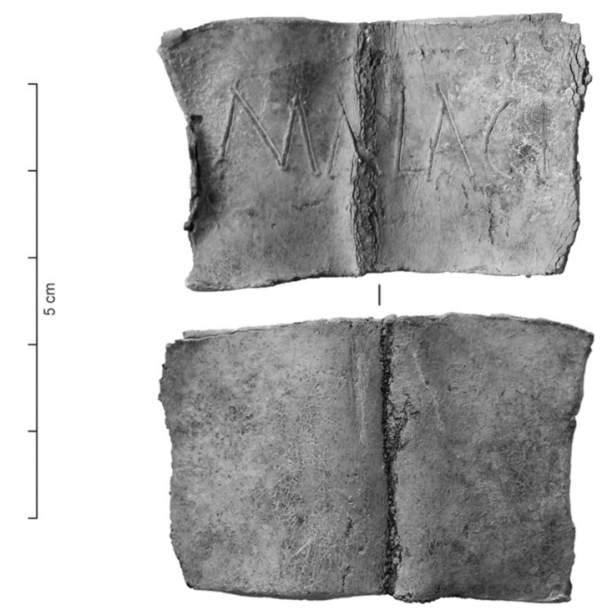

Fig. 4. Etiqueta de plomo de Talairan (Francia). (Foto: Artefacts)

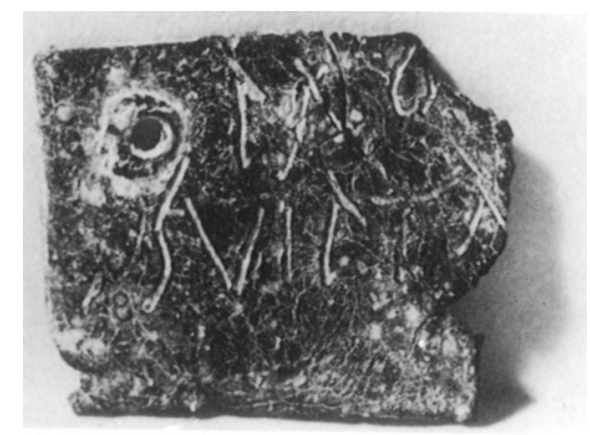

Fig. 5. Etiqueta de plomo de Ampurias. (Foto: IRC)

21 Estas pequeñas plaquitas de plomo fueron ciertamente un modo habitual para etiquetar productos medicinales, pero no el único. Por ejemplo, en Granollers, en el área de Barcelona, apareció una jarra que llevaba incisa la didascalia del producto que contenía, el pericarpium (IRC V, 50, de fin. II - ini. III dC); cf. R. COMES - I. RODÀ (eds.): Scripta manent: La memòria escrita dels romans. Barcelona 2002, 51). 
Se puede concluir, en consecuencia, que la etiqueta de plomo hallada en el Camp de Tarragona debió acompañar un producto preciado, ciertamente elaborado, un preparado galénico cuidadosamente envasado y etiquetado por su productor. Estas características parecen coincidir con el ejemplar de Narbona, a pesar de que desconozcamos de momento la naturaleza del contenido al que aludía el adjetivo malaci. Sin embargo, no es posible de momento ponerla en relación con el ejemplar de Vallmoll hasta que la publicación de nuevos hallazgos permita confirmar en otros ámbitos el uso y función de este tipo de etiquetado especializado.

El profesor Géza Alfödy nos dejó el 6 de noviembre de 2011. Una parte importante de su producción científica y también una parte de su corazón nos lo dejó en Tarragona. Por ello hemos querido participar en este homenaje en recuerdo de su inmensa maestría, que sabía compartir y comunicar como ningún otro gran erudito. Tarragona, y no sólo la Tarraco romana, fue una cirdad que conquistó a Alföldy Géza (en el orden onomástico húngaro). Tuvo con ella, y con todas las personas que se movían en su entorno, una generosidad sin límites, compartiendo sus conocimientos con todos nosotros. Estuvo presente en múltiples actividades desde que empezara la redacción de sus Römische Inschriften von Tarraco, volumen publicado en 1975; pasó por aquel entonces largas temporadas en Tarragona en un apartamento cerca del anfiteatro que siempre recordaba con especial cariño. A partir de 2003, en los inicios del Instituto Catalán de Arqueología Clásica, estuvo apoyando constantemente las diversas iniciativas y el año 2010 estuvo en el acto de constitución del Consejo Científico Asesor del cual era, con toda justicia, miembro nato. El Ayuntamiento de Tarragona y la Universidad Rovira i Virgili le quisieron también demostrar el reconocimiento a su gran labor, otorgándole las máximas distinciones, que aceptó con la humildad de los grandes sabios, dando en cada ocasión lecciones magistrales que quedarán en los anales de la investigación histórica.

La última vez que Géza vino a Tarragona fue el 3 de mayo del 2011. ¡Estaba radiante! Presentó en aquel momento el primero de los tres volúmenes de la editio altera del CIL II referente a Tarraco, una obra ingente que sólo él podía llevar a cabo. En noviembre de 2012, el congreso Tarraco Biennal va a constituir un homenaje internacional a su inmenso legado que se verá aumentado con la presentación del segundo volumen de dicha nueva edición del CIL II y con la de la traducción castellana de la cuarta versión, muy ampliada, de su Historia Social de Roma.

Que estas líneas sean un pequeño pero bien sentido homenaje al maestro que supo ser también amigo. Le echaremos de menos cada día. Sus obras estarán siempre en nuestra compañía; ellas nos seguirán marcando el camino. 
